\title{
Erratum to: Synthesis and characterization of kraft lignin-graft-polylactide copolymers
}

\author{
Seok Ju Kim ${ }^{1}$ Yong Sik Kim ${ }^{1}$ Oh-Kyu Lee ${ }^{1}$ • \\ Byoung-Jun Ahn'
}

\section{Erratum to: Wood Sci Technol DOI 10.1007/s00226-016-0847-8}

In the original publication of the article, Fig. 5 with numbers from 272 to 279 on the right axis is incorrect. The correct Fig. 5 is given below, and the original article has been updated accordingly.

The online version of the original article can be found under doi:10.1007/s00226-016-0847-8.

Yong Sik Kim

yongsikk@korea.kr

1 Division of Wood Chemistry and Microbiology, National Institute of Forest Science, 57 Hoegi-ro, Dongdaemun-gu, Seoul 130-712, Korea 


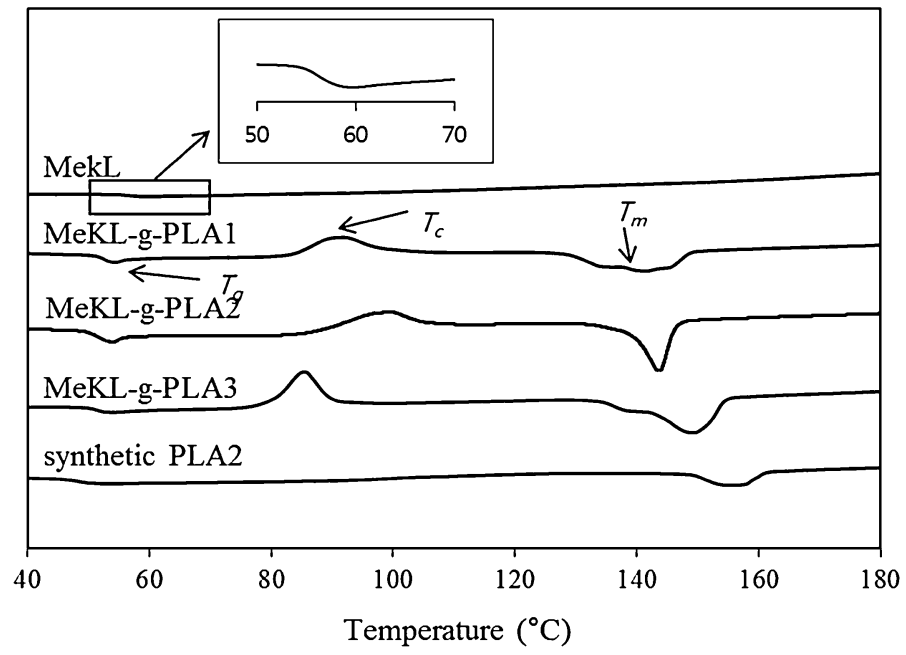

Fig. 5 DSC thermograms of MeKL, synthetic PLA2, and the MeKL-g-PLA copolymers after the third heating scan 